\title{
Coalitional manipulations in a bankruptcy problem
}

\author{
M. Angeles de Frutos
}

Universidad Carlos III de Madrid, Departamento de Economía, Calle Madrid 126, E-28903 Getafe

(Madrid), Spain (e-mail: frutos@eco.uc3m.es)

Received: 24 February 1998 / accepted: 9 March 1999

\begin{abstract}
In a bankruptcy problem framework we consider rules immune to possible manipulations by the creditors involved in the problem via merging or splitting of their individual claims. The paper provides characterization theorems for the non manipulable rules, the no advantageous merging parametric rules and the no advantageous splitting parametric rules.
\end{abstract}

JEL classification: C70, C71, D70

Key words: Axiomatic analysis, merging, splitting

\section{Introduction}

When a firm goes bankrupt, how should its liquidation value be divided among its creditors? A common solution is to allocate the firm's assets proportionally to the creditors' claims or entitlements. There are arguments, however, in favor of other rules. Aumann and Maschler[1], for instance, argue that if the net worth of the firm does not exceed the smallest claim, then equal division among the creditors makes good sense as any claim by one person that goes beyond the entire net worth of the firm might well be considered irrelevant.

The literature devoted to the formal analysis of bankruptcy problems has tried to identify solutions or rules which associate with each bankruptcy problem a

I am indebted to my thesis advisor Jordi Massó for his help, encouragement and understanding during this work. I have also benefited from comments by S. Barberà, D.Moreno, H. Moulin, J. E. Martínez-Legaz, Howard Petith, D. Pérez Castrillo and from suggestions by two anonymous referees. Financial support by a grant from the Generalitat de Catalunya and from DGICYT PB 92-0590 is gratefully acknowledged. 
desirable division between the creditors of the net worth of the firm. ${ }^{1}$ In this paper we study bankruptcy rules that are immune to strategic manipulations whereby a group of creditors merge (i.e., consolidate their claims) in order to represent a single creditor, or a single creditor splits her claim to represent several creditors. We will say that a rule satisfies no advantageous merging (NAM) when no group of creditors is able to benefit by consolidating their claims and being treated as a single creditor. We will say that a rule satisfies no advantageous splitting (NAS) when no creditor is able to benefit by representing several creditors whose claims add up to her. A no advantageous merging and no advantageous splitting rule will be called non manipulable. Non manipulable rules have been studied previously by O'Neill[8] and by Chun[2].

Immunity to these strategic manipulations is relevant in practice as in many bankruptcy problems it is feasible for the creditors to merge or to split. A husband and wife, for example, could present themselves as a single creditor, or the partners of a firm could appear as different creditors. Sometimes, however, it may only be feasible for the creditors to manipulate either by merging or by splitting. Whenever the creditors are just the owners of liabilities issued by a bankrupt firm, manipulation by merging may be possible while manipulations by splitting may not; whereas, if the creditors are banks then manipulations by splitting may enter in a natural way since a bank could divide its claim by transferring it to its subsidiary banks.

In this paper we provide characterization theorems for the rules immune to either kind of these strategic manipulations.

We show that the proportional rule is the only non manipulable bankruptcy rule. For the family of consistent, symmetric and continuous rules (i.e., the parametric rules) we show that the concavity (convexity) of the parametric representation of a rule with respect to the individual claims determines its non manipulability by merging (non manipulability by splitting). Finally, we show that a NAM parametric rule maximizes a strictly concave welfare function where the measure of welfare is the utility derived by the creditors from the award received; whereas a NAS parametric rule minimizes a strictly convex loss function where the measure of loss is the sacrifice that the rule imposes upon the creditors. This result implies that NAM rules are in the spirit of egalitarianism in gains, whereas NAS rules are in the spirit of egalitarianism in losses.

The organization of the paper is as follows. In Sect. 2 we introduce the main concepts and definitions. Section 3 discusses the property of non manipulability. Section 4 contains the characterization results for the NAM and the NAS rules. Finally, the Appendix contains the proofs of the characterization theorems in Sect. 4.

\footnotetext{
${ }^{1}$ For survey on the axiomatic analyses of bankruptcy and taxation problems, see Thomson[12] Notice that the problem of identifying well-behaved taxation rules is formally identical to that of identifying bankruptcy rules, and that all the results for the bankruptcy problem can be reinterprested in the context of taxation
} 


\section{Preliminaries}

We first introduce the class of problems that we study and the necessary notation. Most of the definitions that follow and the notation have been taken from Thomson[12].

A typical bankruptcy problem is that of dividing the net worth $E$ of a bankrupt firm among a group of claimants, $N$, whose claims are specified by a vector $d=\left(d_{i}\right)_{i \in N}$. We denote by $n$ the cardinality of $N$, and by $D$ the total claim. Since we consider situations involving an arbitrary finite number of claimants, it is useful to distinguish between the set of "potential claimants", whose members are identified by a natural number, and the set of claimants actually present in a bankruptcy problem, which we represent by a finite subset $\mathscr{N}$ of the set of natural numbers, $\mathscr{N} \subset \mathbf{N}$.

A Bankruptcy Problem is obtained by first specifying a set of agents $N \in \mathscr{N}$, then a pair $(d ; E) \in \mathbf{R}_{+}^{N} \times \mathbf{R}_{+}$such that $\sum_{i \in N} d_{i} \geq E{ }^{2}$ For $N \in \mathscr{N}$, we denote by $B^{N}$ the class of these problems.

A Bankruptcy Rule $X$ is a function defined on the union of all of the $B^{N}$, for $N \in \mathscr{N}$, which associates with every $N \in \mathscr{N}$ and every $(d ; E) \in B^{N}$ a vector $x \in \mathbf{R}_{+}^{N}: \sum_{i \in N} x_{i}=E$.

Alternatively, a bankruptcy problem can be seen as a problem of dividing a net loss, $D-E$, among the claimants. Nevertheless, the viewpoint we adopt, will affect the allocation, since a given rule may assign gains in a different way than losses. This consideration is related to the concept of dual rule.

Definition 1: Let $X$ be a Bankruptcy rule. Based on $X$ we define its dual $\bar{X}$ in the following way: For all $(d ; E) \in B^{N}, \bar{X}(d ; E)=d-X(d ; D-E) .^{3}$

Note that $\overline{\bar{X}}=X$.

Both rules, $X$ and $\bar{X}$, can be considered as a "philosophy" or as a recommendation about how to undertake the division implicit in every bankruptcy problem. A natural requirement for a rule is that it applies the same "philosophy" regardless of the data (number of creditors, individual claims and net worth of the firm). A way to ensure this is to demand consistency. Consistent rules in this context fulfill a general principle of distributive justice that states that an allocation that is equitable for a group of individuals should be equitable when restricted to a subgroup of individuals. ${ }^{4}$

Definition 2: The rule $X$ satisfies consistency if for all $M, N \subset \mathscr{N}$, for all $(d ; E) \in B^{N}$, if $M \subset N$ and $\left(d_{M} ; \sum_{M} x_{i}\right) \in B^{M}$, where $x=X(d ; E)$, then $\left(x_{i}\right)_{i \in M}=X\left(d_{M} ; \sum_{M} x_{i}\right)$.

\footnotetext{
${ }^{2}$ We denote by $\mathbf{R}^{N}$ the cartesian product of $|N|$ copies of $R$ indexed by the elements of $N$.

${ }^{3}$ Notice that our definition of Bankruptcy rules implies that the dual of a rule $X$ is a well defined rule only if $X$ is claim-bounded (i e, $X_{i}(d ; E) \leq d_{i}$ for all $\left.i \in N\right)$.

${ }^{4}$ Consistency has been a widely used principle in the economic literature in diverse areas, ranging from abstract game theoretic models to concrete taxation and apportionment problems For a review of the role played by the consistency principle in the axiomatic approach to these quite diverse problems, see Thomson [11]
} 
The interpretation of consistency is as follows. Suppose that the rule $X$ assigns allocation $x$ to the bankruptcy problem $(d ; E)$. Suppose, too, that some subset of creditors wants to reallocate the total amount assigned to them. If we apply the same rule to allocate this amount among these creditors, each one will get the amount originally assigned to them, provided $X$ is consistent. So, for example, if $(50,75,100)$ is the recommendation of $X$ to the bankruptcy problem $(100,150,200 ; 225) \in B^{N}$ where $N=\{1,2,3\}$, then $(50,75)$ must be the recommendation of $X$ to the problem $(100,150 ; 125) \in B^{M}$ for $M=\{1,2\}$, provided that $X$ is a consistent rule.

We consider next the possibility that a group of agents may consolidate their claims and be treated as a single claimant, or, conversely, that a given claimant may divide her claim and represent several claimants whose claims add up to her.

Definition 3: A rule $X$ satisfies no advantageous merging if for all $M, N \subset \mathscr{N}$, for all $(d ; E) \in B^{N}$ and $\left(d^{\prime} ; E\right) \in B^{M}$, if $M \subset N$ and there is $i \in M$ such that $d_{i}^{\prime}=d_{i}+\sum_{j \in N \backslash M} d_{j}$ and for all $j \in M \backslash\{i\}, d_{j}^{\prime}=d_{j}$ then $X_{i}\left(d^{\prime} ; E\right) \leq$ $X_{i}(d ; E)+\sum_{j \in N \backslash M} X_{j}(d ; E)$.

Here no possible coalition of creditors has an incentive to pool their claims to be treated as a single creditor whose claim is the sum of the individual claims of their constituents.

Definition 4: A rule $X$ satisfies no advantageous splitting if for all $M, N \subset \mathscr{N}$, for all $(d ; E) \in B^{N}$ and $\left(d^{\prime} ; E\right) \in B^{M}$, if $M \subset N$ and there is $i \in M$ such that $d_{i}^{\prime}=d_{i}+\sum_{j \in N \backslash M} d_{j}$ and for all $j \in M \backslash\{i\}, d_{j}^{\prime}=d_{j}$ then $X_{i}\left(d^{\prime} ; E\right) \geq$ $X_{i}(d ; E)+\sum_{j \in N \backslash M} X_{j}(d ; E)$.

Namely, no claimant has an incentive to divide her claim to represent several creditors whose claims add up to her.

Definition 5: A rule $X$ is called non manipulable if it satisfies no advantageous splitting and no advantageous merging.

Whenever a rule satisfies no advantageous merging it is called a NAM rule, whereas if it satisfies no advantageous splitting it is called a NAS rule.

Proposition 1 shows that there is a dual relation between the family of no advantageous splitting rules, $\mathscr{S}$, and the family of no advantageous merging ones, $\mathscr{M}$.

Proposition 1. If $X$ is a bankruptcy rule such that its dual $\bar{X}$ is well defined, then $X \in \mathscr{M}$ if and only if $\bar{X} \in \mathscr{S}$.

Proof. The proof of the statement requires to prove:

a) If $X \in \mathscr{M}$ and $\bar{X}$ is well defined then $\bar{X} \in \mathscr{S}$, and

b) If $\bar{X}$ is well defined and $\bar{X} \in \mathscr{T}$ then $X \in \mathscr{M}$.

We first show (a) by way of contradiction. Assume $X \in \mathscr{U}$ and $\bar{X}$ is well defined but $\bar{X} \notin \mathscr{S}$. Since $\bar{X} \notin \mathscr{S}$ there exist $(d ; E) \in B^{N}$ and $\left(d^{\prime} ; E\right) \in B^{M}, N \supset M$, 
and there is $i \in M$ with $d_{i}^{\prime}=d_{i}+\sum_{j \in N \backslash M} d_{j}$ and with $d_{j}^{\prime}=d_{j}$ for all $j \in M$ $\backslash\{i\}$, for which

$$
\bar{X}_{i}\left(d^{\prime} ; E\right)<\bar{X}_{i}(d ; E)+\sum_{j \in N \backslash M} \bar{X}_{j}(d ; E)
$$

Applying the definition of dual rule (1) can be rewritten as follows:

$d_{i}^{\prime}-X_{i}\left(d^{\prime} ; D^{\prime}-E\right)<d_{i}-X_{i}(d ; D-E)+\sum_{j \in N \backslash M} d_{j}-\sum_{j \in N \backslash M} X_{j}(d ; D-E)$

Since $d_{i}^{\prime}=d_{i}+\sum_{j \in N \backslash M} d_{j}$ and since $D^{\prime}=D$ then (2) becomes

$$
X_{i}\left(d^{\prime} ; D-E\right)>X_{i}(d ; D-E)+\sum_{j \in N \backslash M} X_{j}(d ; D-E) .
$$

The above result contradicts $X \in \mathscr{M}$ since it is advantageous for the members of $T$ ( $T=N \backslash M \cup\{i\}$ ) to merge their claims to be treated as a single creditor. Thus if $X \in \mathscr{M}$ and $\bar{X}$ is well defined then $\bar{X} \in \mathscr{S}$. The proof of the statement: If $X \in \mathscr{S}$ and $\bar{X}$ is well defined then $\bar{X} \in \mathscr{M}$, is similar to the previous one and therefore it is omitted.

Now to prove b) let $\bar{X}$ be a well defined rule such that $\bar{X} \in \mathscr{S}$. Since $\overline{\bar{X}} \in \mathscr{M}$ and $\overline{\bar{X}}=X$ we have that $X \in \mathscr{M}$ as we claimed.

To illustrate the strategic properties defined earlier we now apply them to the Talmudic rule. This rule was first studied by Aumann and Maschler. The definition that follows is taken from Thomson[12].

The Talmudic rule: For all $(d ; E) \in B^{N}$ and for all $i \in N$,

-If $E \leq D / 2$, then $x_{i}=\min \left\{\lambda, d_{i} / 2\right\}$, where $\lambda$ is chosen so that $\sum_{i \in N} \min \left\{\lambda, d_{i} / 2\right\}=E$,

-If $E \geq D / 2$, then $x_{i}=d_{i}-\min \left\{\lambda, d_{i} / 2\right\}$, where $\lambda$ is chosen so that $\sum_{i \in N} \min \left\{\lambda, d_{i} / 2\right\}=D-E$.

Notice that for bankruptcy problems in which $E \leq D / 2$ the Talmudic rule can be regarded as a special case of the Constrained-Equal-Award rule; and for those problems in which $E \geq D / 2$, it is a special case of the Constrained-Equal-Losses rule.

The Talmudic rule is an example of a rule that it is manipulable by splitting and by merging. Moreover, it can be shown that whenever the net worth of the firm is smaller than half the total claim the Talmudic rule is manipulable by splitting and not by merging, while the opposite holds whenever the net worth of the firm is greater than half the total claim. Section 4 provides a formal proof of this statement. Here we only give numerical examples to illustrate the above statement.

Consider the problem $(100,200,300,400 ; 400) \in B^{N}$ where $E=400<D / 2=$ 500 , and $N=\{1,2,3,4\}$. The Talmudic shares are $(50,100,125,125)$. Suppose that creditor 4 with a claim of 400 divides her claim into two equal claims to represent creditors 4 and 5 in the bankruptcy problem $(100,200,300,200,200 ; 400)$ 
$\in B^{M}$ where $M=\{1,2,3,4,5\}$. The shares are now $(50,87.5,87.5,87.5,87.5)$. That creditor gets now $87.5+87.5=175>125$, so she has incentives to manipulate by splitting.

Consider now the problem $(100,200,300,400 ; 700) \in B^{N}$ where $E=700>$ $D / 2=500$ and $N=\{1,2,3,4\}$. The Talmudic shares are $(50,116.6,216.6$, 316.6). If creditors 1 and 2 merge their claims to be represented by creditor 1 in the bankruptcy problem $(300,300,400 ; 700) \in B^{P}$ where $P=\{1,3,4\}$ the shares will be $(200,200,300)$. Since $200>50+116.6$ creditors 1 and 2 have incentives to manipulate by merging.

\section{Non manipulable rules}

In the previous section we showed that the Talmudic rule is a manipulable rule. The result obtained for the Talmudic rule can be expanded to the egalitarian rules of which that is a special case. It is also known, from the work by O'Neill[8], that there is at least a rule whereby the creditors have no incentive to either merge or split their individual claims. O'Neill provided a characterization of the proportional rule as the only rule that satisfies (1) symmetry, (2) continuity at at least one point, (3) independency of the addition of dummy creditors with zero claim and (4) strategy-proofness. Strategy-proofness is equivalent to non manipulability, as we mentioned earlier. A natural question arises from this result: Is there some other non manipulable rule if (1), (2) and (3) are not required? We provide a negative answer to this question through a characterization theorem that states that the proportional rule is the only rule that satisfies non manipulability for all possible bankruptcy problems.

We will devote this section to study the implications of non-manipulability. Let us recall that non manipulable rules are those satisfying no advantageous splitting and no advantageous merging. Thus if $X$ is a non manipulable rule then for all $M, N \subset \mathscr{N}$, for all $(d ; E) \in B^{N}$ and $\left(d^{\prime} ; E\right) \in B^{M}$, if $M \subset N$ and there is $k \in M$ such that $d_{k}^{\prime}=d_{k}+\sum_{j \in N \backslash M} d_{j}$ with $d_{j}^{\prime}=d_{j}$ for all $j \in M \backslash\{k\}$, then $X_{k}(d ; E)+\sum_{j \in N \backslash M} X_{j}(d ; E)=X_{k}\left(d^{\prime} ; E\right)$.

An immediate consequence of the above remark (see next proposition) is that non manipulable rules assign to each creditor a share that only depends upon the total claim, the net worth of the bankrupt firm and her own claim.

Proposition 2. If the rule $X$ satisfies non manipulability, then for all $N \in \mathscr{N}$, $(d ; E) \in B^{N}$ and for all $i \in N$, if $d^{\prime}=\left(d_{i}, d_{k}\right)$ where $d_{k}=D-d_{i}$ and $k \in N \backslash\{i\}$ then $X_{i}(d ; E)=X_{i}\left(d_{i}, D-d_{i} ; E\right)$.

The proof follows immediately from the definition of non manipulable rules. Consider $(d ; E) \in B^{N}$ and $\left(d^{\prime} ; E\right) \in B^{M}$ such that $M \subset N$ with $M=\{i, k\}$, with $d_{k}^{\prime}=D-d_{i}$ and with $d_{i}^{\prime}=d_{i}$. By non-manipulability $X_{k}(d ; E)=X_{k}\left(d^{\prime} ; E\right)=$ $X_{k}\left(d_{i}, D-d_{i} ; E\right)$. By budget balance we have that $X_{i}(d ; E)=X_{i}\left(d_{i}, D-d_{i} ; E\right)$.

Notice that when a set of creditors (a coalition $T \subset N$ ) merge their claims they will be represented by one of the members of the coalition who will show a 
claim equal to the sum of the claims of the creditors in the coalition. Therefore, the coalition can choose among its members who will represent it. Similarly, when a creditor $i \in N$ facing the problem $(d ; E) \in B^{N}$ decides to split her claim into, for instance, two parts, she will then represent herself, $i$, and another creditor $j$. She can choose any name for $j$ ( any natural number) provided that $j$ is not in $N$. Because of our definition of mergers and splitting, non manipulable rules will assign shares that do not depend upon the names of the creditors. ${ }^{5}$

We now show that non manipulable rules satisfy symmetry, the dummy axiom, and order preservation.

Proposition 3. Every non manipulable rule satisfies symmetry, that is, for all $N \in \mathscr{N}$, all $(d ; E) \in B^{N}$ and all $i \in N$, if $d_{i}=d_{j}$ then $X_{i}(d ; E)=X_{j}(d ; E)$.

Proof. Assume, by way of contradiction, that $X$ is a non manipulable rule that violates symmetry, that is, there exist $N \in \mathscr{N}$ and $(d ; E) \in B^{N}$, with $d_{i}=d_{j}=\bar{d}$ for some $i, j \in N$, but with $X_{i}(d ; E)>X_{j}(d ; E)$. Consider first the case $n \geq 3$. Let $k \in N, k \neq i, j$.

Assume that all creditors in $N$ but $j$ merge their claims under the name of $k$. The resulting bankruptcy problem will be $\left(d^{\prime} ; E\right) \in B^{M}$ with $M=\{j, k\}$, such that $d_{k}^{\prime}=D-\bar{d}$ and $d_{j}^{\prime}=d_{j}=\bar{d}$. Clearly, if $X_{k}\left(d^{\prime} ; E\right) \neq E-X_{j}(d ; E)$ then $X$ is manipulable. Thus $X_{k}\left(d^{\prime} ; E\right)=E-X_{j}(d ; E)$. Let us assume now that all creditors in $N$ but $i$ facing the problem $(d ; E) \in B^{N}$ decide to merge their claims under the name of $k$. The resulting bankruptcy problem will be $\left(d^{\prime \prime} ; E\right) \in B^{P}$, $P=\{i, k\}$, with $d_{k}^{\prime \prime}=D-\bar{d}$ and $d_{i}^{\prime \prime}=d_{i}=\bar{d}$. By non manipulability,

$$
X_{k}\left(d^{\prime \prime} ; E\right)=E-X_{i}(d ; E)<E-X_{j}(d ; E)=X_{k}\left(d^{\prime} ; E\right)
$$

Because of budget balance, $X_{i}\left(d^{\prime \prime} ; E\right)>X_{j}\left(d^{\prime} ; E\right)$. We now show that $X$ is manipulable by splitting. Assume creditor $i$ facing the problem $\left(d^{\prime \prime} ; E\right)$ divides her claim into two equal claims to represent creditor $i$ with claim $\bar{d} / 2$ and creditor $j$ with claim $\bar{d} / 2$ in the problem $(\widetilde{d} ; E) \in B^{T}$ where $T=\{i, j, k\}$. Because of non manipulability $X_{i}\left(d^{\prime \prime} ; E\right)=X_{i}(\widetilde{d} ; E)+X_{j}(\widetilde{d} ; E)$. Assume now that creditor $j$ facing the problem $\left(d^{\prime} ; E\right)$ divides her claim into two equal claims to represent creditor $i$ with claim $\bar{d} / 2$ and creditor $j$ with claim $\bar{d} / 2$ in the problem $(\widetilde{d} ; E) \in B^{T}$. Since $X_{i}\left(d^{\prime \prime} ; E\right)=X_{i}(\widetilde{d} ; E)+X_{j}(\widetilde{d} ; E)>X_{j}\left(d^{\prime} ; E\right)$, we can conclude that $X$ is manipulable by splitting, a contradiction.

Consider now the case $n=2$. Let $(d ; E) \in B^{N}$ be a bankruptcy problem such that $d_{1}=d_{2}$. Suppose both creditors divide their claims into two equal claims. Now symmetry for $n=3$ plus non-manipulability implies symmetry for $n=2$.

\footnotetext{
${ }^{5}$ To illustrate this statement consider the rule $X$ that gives all the net worth of the bankrupty firm to the claimant with the smallest $i$ (i e $X_{i}(d ; E)=E$ if $i=\min _{j \in N} j$ and $X_{i}(d ; E)=0$ otherwise) Thus creditor 3 facing the problem $(d ; E) \in B^{N}$ with $N=\{2,3,4\} \in \mathscr{N}$ will get a zero payment If this creditor divides her claim into two equal claims to represent creditor 1 and creditor 3 in the bankruptcy problem $\left(d^{\prime} ; E\right) \in B^{M}$ with $M=\{1,2,3,4\}$ and with $d_{j}^{\prime}=d_{j}$ for all $j \in M \backslash\{3\}$, then $X_{1}\left(d^{\prime} ; E\right)+X_{3}\left(d^{\prime} ; E\right)=E>X_{3}(d ; E)=0$. Hence $X$ will be manipulable by splitting
} 
Proposition 4. Every non manipulable rule $X$ satisfies the dummy axiom, that is, for all $N \in \mathscr{N},(d ; E) \in B^{N}$ and for all $i \in N$, if $d_{i}=0$ then $X_{i}(d ; E)=0$.

The proof is immediate. It follows from the fact that if it does not hold then there exists some creditor who has incentives to split her claim into her claim and some zero claims to get a better share.

Proposition 5. If a rule is non manipulable then it satisfies order preservation, i.e., for all $N \in \mathscr{N},(d ; E) \in B^{N}$ and for all $i \in N$, if $d_{i}<d_{j}$ then $X_{i}(d ; E)<$ $X_{j}(d ; E)$.

Proof. Let $N \in \mathscr{N}$ and $(d ; E) \in B^{N}$, such that there exist $i$ and $j$ in $N$ with $d_{i}<d_{j}$.

Assume that creditor $j$ divides her claim to represent creditor $j$ with claim $d_{i}$ and creditor $k, k \notin N$, with claim $d_{j}-d_{i}$ in the bankruptcy problem $\left(d^{\prime} ; E\right) \in B^{M}$ where $M=N \cup\{k\}$ and $d_{s}=d_{s}^{\prime}$ for all $s \in N \backslash\{j\}$. Symmetry implies $X_{j}\left(d^{\prime} ; E\right)=$ $X_{i}\left(d^{\prime} ; E\right)$ and non-manipulability implies $X_{i}\left(d^{\prime} ; E\right)=X_{i}(d ; E)$. Therefore

$$
X_{j}(d ; E)=X_{j}\left(d^{\prime} ; E\right)+X_{k}\left(d^{\prime} ; E\right)=X_{i}(d ; E)+X_{k}\left(d^{\prime} ; E\right) \geq X_{i}(d ; E) .
$$

We now show that $X_{j}(d ; E)>X_{i}(d ; E)$ by proving that $X_{k}\left(d^{\prime} ; E\right)>0$.

Consider the bankruptcy problem $\left(d^{\prime \prime} ; E\right) \in B^{P}$ such that $P=\{i, k\}, d_{i}^{\prime \prime}=D-d_{k}^{\prime}$ and $d_{k}^{\prime \prime}=d_{k}^{\prime}=d_{j}-d_{i}$. Appealing to Proposition 1 we know that

$$
X_{k}\left(d^{\prime} ; E\right)=X_{k}\left(d_{i}^{\prime \prime}, d_{k}^{\prime \prime} ; E\right)=X_{k}\left(D-d_{j}+d_{i}, d_{j}-d_{i}, ; E\right) .
$$

Let us assume first that $\frac{D-d_{j}+d_{i}}{d_{j}-d_{i}} \in \mathrm{N}$. If creditor $i$ facing the problem $\left(d^{\prime \prime} ; E\right)$ divides her claim into $\frac{D-d_{j}+d_{i}}{d_{j}-d_{i}}$ equal claims to represent $\frac{D-d_{j}+d_{i}}{d_{j}-d_{i}}$ creditors each with a claim of $d_{j}-d_{i}$ then non-manipulability (and consequently symmetry) plus budget balance yield $X_{k}\left(d^{\prime} ; E\right)>0$.

Assume now that $\frac{D-d_{j}+d_{i}}{d_{j}-d_{i}} \notin \mathrm{N}$. Let us denote by $t$ to the maximum integer smallest than $\frac{D-d_{j}+d_{i}}{d_{j}-d_{i}}$. If creditor $i$ divides her claim to represent $t+1$ creditors, $t$ of them with a claim of $d_{j}-d_{i}$ and the remaining creditor with a claim of $D-(t+1)\left(d_{j}-d_{i}\right)$, then this splitting yields a bankruptcy problem $(\widetilde{d} ; E) \in B^{R}$ with $P \subset R$ in which $\widetilde{d}_{s} \leq d_{j}-d_{i}$ for all $s \in R$. Let us assume, w.1.o.g. that $\widetilde{d}_{2}=D-(t+1)\left(d_{j}-d_{i}\right)$. By non manipulability $X_{k}\left(d_{i}^{\prime \prime}, d_{k}^{\prime \prime} ; E\right)=X_{k}(\widetilde{d} ; E)$. Because of symmetry $X_{k}(\tilde{d} ; E)=X_{s}(\tilde{d} ; E)$ for all $s \in R \backslash\{2\}$, with $X_{k}(\widetilde{d} ; E) \geq X_{2}(\tilde{d} ; E)$ since $\widetilde{d}_{k}=d_{j}-d_{i}>\widetilde{d}_{2}$. These results plus budget balance imply $X_{k}\left(d^{\prime} ; E\right)=$ $X_{k}(\widetilde{d} ; E)>0$.

Theorem 1. There is exactly one non manipulable bankruptcy rule: the proportional rule.

Proof. It is known that the proportional rule is non manipulable. The proof of the converse statement is done in two steps. 
Step 1. We first show that for all $N \in \mathscr{N}$, and for all $(d ; E) \in B^{N}$, a non manipulable rule assigns a share of $s E / t$ to any individual claim of $s D / t$, for any integers $s$ and $t$ such that $0 \leq s \leq t$.

Let $(d ; E)$ be a bankruptcy problem such that $d_{i}=s D / t$ for some $i \in N$. Without loss of generality let $i=1$. Since $D-d_{1}=(t-s) D / t$, Proposition 1 implies that

$$
X_{1}(d ; E)=X_{1}(s D / t,(t-s) D / t ; E) .
$$

Let us denote by $d^{\prime}$ to the vector $(s D / t,(t-s) D / t)$. Because of non manipulability if creditor with claim $(t-s) D / t$ facing the problem $\left(d^{\prime} ; E\right)$ presents herself as $(t-s)$ creditors each with a claim of $D / t$ she will not gain by doing so. Therefore

$$
X_{1}(s D / t,(t-s) D / t ; E)=X_{1}\left(d^{\prime \prime} ; E\right)
$$

where $d_{1}^{\prime \prime}=d_{1}^{\prime}=s D / t$ and $d_{j}^{\prime \prime}=D / t$ for all $j \neq 1$. Without loss of generality let $j=2, \ldots, t-s+1$. Assume now that creditor 1 facing the problem $\left(d^{\prime \prime} ; E\right)$ splits her claim into $s$ equal claims of $D / t$ to represent creditors $1, t-s+2, \ldots, t$ in the bankruptcy problem $(\widetilde{d}, E) \in B^{M}$ where $\widetilde{d}_{i}=D / t$ for all $i \in M$. Notice that $\widetilde{d}_{1}+\sum_{i=t-s+2}^{t} \widetilde{d}_{i}=d_{1}^{\prime \prime}$ and $d_{j}^{\prime \prime}=\widetilde{d}_{j}$ for all $j=2, \ldots, t-s+1$. Appealing to non manipulability by splitting we have that

$$
X_{1}\left(d^{\prime \prime} ; E\right)=X_{1}(\widetilde{d} ; E)+\sum_{i=t-s+2}^{t} X_{i}(\tilde{d} ; E)
$$

Symmetry implies that the right hand side of (5) is $s E / t$. Thus (3), (4) and (5) imply that $X_{1}(d ; E)=s E / t$.

Step 2. We now show that for any $N \in \mathscr{N}$ and $(d ; E) \in B^{N}$ any non manipulable rule assigns $d_{i} E / D$ to any claim of $d_{i}$, for any $i \in N$.

It is well known that for any value of $\left(d_{i} / D\right) \in[0,1]$, and for every $K \geq 1$, there exists some integer ${ }^{6} p_{K}, 0 \leq p_{K} \leq 2^{K}$, such that: $\frac{p_{K}}{2^{K}} \leq \frac{d_{i}}{D} \leq \frac{1+p_{K}}{2^{K}}$. Thus for any $N \in \mathscr{N}$ and $(d ; E) \in B^{N}$ and for any $i \in N$ the following inequality holds:

$$
\frac{D p_{K}}{2^{K}} \leq d_{i} \leq \frac{D\left(1+p_{K}\right)}{2^{K}} .
$$

Proposition 1 implies that $X_{i}(d, E)=X_{i}\left(d_{i}, D-d_{i}, E\right)=X_{i}\left(d^{\prime}, E\right)$ whenever $d^{\prime}$ be such that $d_{k}=d_{k}^{\prime}$ for all $k \in N, k \neq j, l, d_{j}^{\prime}=p_{K} D / 2^{K}$ and $d_{l}^{\prime}=d_{l}+d_{j}-p_{K} D / 2^{K}$. Notice that $d^{\prime}=d$ if $d_{j}=p_{K} D / 2^{K}$. Since $d_{j}^{\prime}=\frac{D p_{K}}{2^{K}} \leq d_{i}=d_{i}^{\prime}$ order preservation and symmetry imply $X_{j}\left(d^{\prime}, E\right) \leq X_{i}\left(d^{\prime}, E\right)$. Because of result in step 1 (take $s=p_{K}$ and $t=2^{K}$ ) we know that $X_{j}\left(d^{\prime}, E\right)=p_{K} E / 2^{K}$. Therefore $p_{K} E / 2^{K} \leq X_{i}\left(d^{\prime}, E\right)$.

Similarly, by appealing again to proposition 1 , we know that $X_{i}(d, E)=$ $X_{i}\left(d_{i}, D-d_{i}, E\right)=X_{i}\left(d^{\prime \prime}, E\right)$ for $d^{\prime \prime}$ such that $d_{k}=d_{k}^{\prime \prime}$ for all $k \in N, k \neq j, l$, $d_{j}^{\prime}=\left(1+p_{K}\right) D / 2^{K}$ and $d_{l}^{\prime}=d_{l}+d_{j}-\left(1+p_{K}\right) D / 2^{K}$. Result in step 1 yields

\footnotetext{
${ }^{6} p_{K}$ is nothing but the integer part of $\left(2^{K} \frac{d_{i}}{D}\right)$. We write $\frac{d_{i}}{D}$ in binary representation
} 
$X_{j}\left(d^{\prime \prime}, E\right)=\left(1+p_{K}\right) E / 2^{K}$. Since $d_{i}^{\prime \prime}=d_{i}^{\prime} \leq d_{j}^{\prime \prime}$ order preservation and symmetry imply

$$
\frac{E p_{K}}{2^{K}} \leq X_{i}(d, E) \leq \frac{E\left(p_{K}+1\right)}{2^{K}} .
$$

The sequence $p_{K} / 2^{K}$ is bounded and increasing in $K$, whereas the sequence $\left(1+p_{K}\right) / 2^{K}$ is bounded and decreasing in $K$. As $K \rightarrow \infty$ they converge to $d_{i} / D$. The limiting relation $\frac{d_{i} E}{D} \leq X_{i}(d, E) \leq \frac{E d_{i}}{D}$ implies that a rule is non manipulable if and only if gives proportional shares. $\square$

Let us now comment on the related work by Chun[2]. Chun characterizes the proportional rule by imposing symmetry, continuity, Non advantageous reallocation (NAR, for short) and the dummy axiom. NAR says that no coalition can benefit from redistributing the claims among its members. Chun also shows that the dummy axiom in O'Neill's result is redundant and that NAR and the dummy axiom imply strategy-proofness. Clearly, a non manipulable rule satisfies NAR, while the converse does not hold (think of the egalitarian in gains rules that satisfy NAR but are manipulable by splitting). Hence the main difference between Theorem 1 and Chun's earlier results is that Theorem 1 shows that continuity and symmetry are no longer required to characterize the proportional rule.

\section{No advantageous merging and no advantageous splitting rules}

Theorem 1 tells us that to require non manipulability is equivalent to require proportional division. One could argue that this property is too demanding because it rules out strategic manipulations that may, for some bankruptcy problems, not be feasible. This consideration is particularly relevant in the context of income taxation. There only manipulations by merging are possible -when the married taxpayers choose between filing a joint return or filing an individual return- but manipulations by splitting are not possible. This consideration has brought us to search for bankruptcy rules that satisfy either no advantageous merging or no advantageous splitting. Among all possible bankruptcy rules we focus on those that satisfy consistency. Consistency seems to us a natural requirement to impose on any rule. About this property W. Thomson[11] writes : "Consistency says that if some of the claimants leave with their awards, and the situation is reevaluated from the viewpoint of the remaining claimants, the solution should assign to them the same awards as initially".

The implications of consistency has been described very completely, with very few auxiliary conditions. Consider indeed the following class of rules introduced by Young (1987).

Parametric rules. Let $h:[a, b] \times \mathscr{R}_{+} \rightarrow \mathscr{R}_{+}$, where $[a, b] \subset[-\infty,+\infty]$, be a function that is a continuous, is (weakly) monotonic in its first argument, and satisfies $h\left(a, d_{i}\right)=0$ and $h\left(b, d_{i}\right)=d_{i}$ for all $d_{i} \in \mathscr{R}_{+}$. Then, given $N \in \mathscr{N}$ 
and $(d ; E) \in B^{N}$, the parametric rule relative to $h$ selects the point $x \in \mathscr{R}^{N}$ such that for some $\lambda \in[a, b], \sum_{i \in N} x_{i}=E$ and $x_{i}=h\left(\lambda, d_{i}\right)$ for all $i \in N .^{7}$

Young [13] has shown that a continuous rule is symmetric and consistent if and only if it is a parametric rule. This section is devoted to study the parametric rules that satisfy either non manipulablity by merging or non manipulability by splitting.

Next theorem which builds in the work by Young shows that two interesting subfamilies of the parametric family can be identified by imposing the requirement of no advantageous merging or the requirement of no advantageous splitting.

Theorem 2. a). A continuous, symmetric and consistent rule is non manipulable by splitting, if and only if it is a parametric rule relative to a function $h$ that is convex in its second argument for each value of the parameter $\lambda$.

$b$ ). It is non manipulable by merging, if and only if it is a parametric rule relative to a function $h$ that is concave in its second argument for each value of the parameter $\lambda$.

\section{Proof. See Appendix.}

Remark 1. A continuous, symmetric and consistent rule is non manipulable if and only if it is a parametric rule relative to a function $h$ that is linear in its second argument for each value of the parameter $\lambda$.

In light of Theorem 2 we can now easily study whether some of the most wellknown parametric rules are non manipulable by splitting and/or non manipulable by merging. Consider the following parametric rules:

$$
\begin{aligned}
& h\left(\lambda, d_{i}\right)=\min \left\{\lambda, d_{i}\right\}, 0 \leq \lambda \leq \infty \text { (Constrained-Equal-Award rule), } \\
& h\left(\lambda, d_{i}\right)=\max \left\{0, d_{i}-1 / \lambda\right\}, 0 \leq \lambda \leq \infty \text { (Constrained-Equal-Losses rule) } \\
& h\left(\lambda, d_{i}\right)=\lambda d_{i}, 0 \leq \lambda \leq 1 \text { (Proportional rule). }
\end{aligned}
$$

The Constrained-Equal-Award rule is non manipulable by merging since its parametric representation is concave in $d_{i}$, whereas the Constrained-Equal-Losses rule is non manipulable by splitting. The proportional rule is non manipulable since its parametric representation is linear in $d_{i}$. Finally, the Talmudic rule is non manipulable by merging if and only if $E \leq D / 2$, since only in that case its parametric representation satisfies concavity in its second argument (recall that its parametric representation in that case is $\left.h\left(\lambda, d_{i}\right)=\min \left\{\lambda, d_{i} / 2\right\}\right)$, whereas if $E \geq$ $D / 2$, it is a non manipulable by merging rule since its parametric representation is $h\left(\lambda, d_{i}\right)=\max \left\{0, d_{i} / 2-1 / \lambda\right\}$.

\footnotetext{
${ }^{7}$ Notice that the function $h\left(\lambda, d_{i}\right)=\lambda d_{i}^{2}$ does not represent a parametric rule since it does not exist $b$ such that $h\left(b, d_{i}\right)=d_{i}$ for all $d_{i} \in \mathscr{R}_{+}$. By construction of $h$ it is deduced that a parametric rule always satisfies Claim-boundness (i e , $\left.0 \leq h\left(\lambda, d_{i}\right) \leq d_{i}\right)$. Thus, a parametric rule satisfies the dummy axiom It is also deduced from the definition of $h$ that, as $\lambda$ rises from $a$ to $b$, the function $\sum_{i \in N} h\left(\lambda, d_{i}\right)$ continuously increases (or is constant) from 0 to $\sum_{i \in N} d_{i}$ Therefore the equation $\sum_{i \in N} h\left(\lambda, d_{i}\right)=E$ has a solution if and only if $\sum_{i \in N} d_{i} \geq E$ It may have several solutions if all functions $h\left(\lambda, d_{i}\right)$ are flat in $\lambda$ at the same time, but the vector of shares is always unique
} 
It is also known from Young's work that a parametric rule $h$ can be obtained by minimizing a symmetric, continuous and additively separable loss function $\sum_{i \in N} H\left(x_{i}, d_{i}\right)$ over the constraint set $\left\{x \in \mathscr{R}^{n}: \sum_{i=1}^{n} x_{i}=E, 0 \leq x_{i} \leq d_{i}\right\}$, where $H(.,$.$) is a strictly convex function. The relationship between h$ and $H$ is the following: $H\left(x_{i}, d_{i}\right)=\int_{0}^{x_{i}} f\left(a, d_{i}\right) d a$, where $f$ is any pseudo-inverse of $h{ }^{8}$ From this approach we now show that any NAM parametric rule minimizes a convex loss function that only depends on the amount received $\left(H\left(x_{i}, d_{i}\right)=\right.$ $\left.U\left(x_{i}\right)\right)$, while any NAS parametric rule minimizes a convex loss function that depends on the sacrifice imposed on the creditors $\left(H\left(x_{i}, d_{i}\right)=U\left(d_{i}\right)-U\left(d_{i}-x_{i}\right)\right)$. One may think of $U($.$) and V($.$) as utility functions that apply to all individuals.$ Thus, any NAM parametric rule is somehow egalitarian in gains, whereas any NAS parametric rule is somehow egalitarian in losses.

Theorem 3. a). A parametric rule satisfies no advantageous splitting if and only if its solutions minimize a symmetric, continuous, additively separable, strictly convex objective function that takes the form $H\left(x_{i}, d_{i}\right)=U\left(d_{i}\right)-U\left(d_{i}-x_{i}\right)$ for any no advantageous splitting parametric rule but the proportional rule for which $H\left(x_{i}, d_{i}\right)=x_{i}^{2} / 2 d_{i}$.

$b)$. It satisfies no advantageous joining if and only if the objective function takes the form $H\left(x_{i}, d_{i}\right)=U\left(x_{i}\right)$ for any no advantageous merging parametric rule but the proportional rule for which $H\left(x_{i}, d_{i}\right)=x_{i}^{2} / 2 d_{i}$.

Proof. See Appendix.

\section{Conclusion}

In this paper we have proposed three axioms with a strategic flavor: non manipulability, non advantageous merging and non advantageous splitting. We have shown that the combination of the axiom of consistency with either NAM or NAS characterizes a family of rules that always include the proportional one. Moreover the shares of any consistent and NAM rule will go from the uniform in gains (the most egalitarian in gains) to the proportional ones (the least egalitarian), and, analogously, the shares of any consistent and NAS rule will go from proportional (the least egalitarian in losses) to the uniform in losses (the most egalitarian).

Finally we comment on related work in the literature. Three well known axioms are related to those in this paper: Strategy-proofness, non advantageous reallocation and composition. As we mentioned in Sect. 3, any strategy-proofness rule is non manipulable. Any non manipulable rule satisfies NAR, furthermore, they are a subset of the NAM ones, while they are manipulable via divisions. Finally, in Young [14] a characterization based mainly in the axiom of composition is provided. Composition says that every increment of the estate should be

\footnotetext{
${ }^{8}$ Whenever $h$ is an strictly monotone increasing in $\lambda$ function, $f$ will be the inverse of $h$. Notice that $f$ is not necessarily continuous Nevertheless, for fixed $d_{i} \geq 0, f\left(x_{i}, d_{i}\right)$ is monotone increasing in $x_{i}$, hence the set of points $x_{i}$ where $f$ is discontinuous is countable
} 
assessed equitably relative to the creditor's current loss. Young has shown that a strictly monotonic, strictly order-preserving rule satisfies consistency, composition and scale invariance if and only if it equalizes absolute sacrifice. We have here shown that any rule in this family is a NAS mechanism.

\section{Appendix: proofs}

Notation. Throughout this appendix we denote by $\Lambda(d)$ the set of values that solve the equation $\sum_{i \in N} h\left(\lambda, d_{i}\right)=E$ for the problem $(d ; E) \in B^{N}, N \in \mathscr{N}$. Each $\Lambda(\cdot)$ is always non empty and closed (recall that $h$ is a continuous function). $\Lambda(\cdot)$ is compact if the closed interval on which $\lambda$ is defined is compact. It will be a singleton whenever $h$ is strictly monotonic in $\lambda$.

We now rewrite the property of NAS for a parametric rule. The NAM property is similar.

A parametric rule is non manipulable by splitting if for all $(d ; E) \in B^{N}$ and $\left(d^{\prime}, E\right) \in B^{M}, M \subset N$ if there is $i \in M$ with $d_{i}^{\prime}=d_{i}+\sum_{j \in N \backslash M} d_{j}$ and with $d_{j}^{\prime}=d_{j}$ for all $j \in M \backslash\{i\}$, then $\lambda \geq \lambda^{\prime}$ for any $\lambda \in \Lambda(d)$ and $\lambda^{\prime} \in \Lambda\left(d^{\prime}\right)$. Notice that in order to ensure that creditor $i$ has no incentive to split her claim to represent $(\# N / M)+1$ creditors, $h\left(\lambda, d_{j}\right) \geq h\left(\lambda^{\prime}, d_{j}^{\prime}\right)$ must hold for all $j \in M$ $\backslash\{i\}$. It is deduced from this definition that a NAS parametric rule satisfies, for fixed $\lambda$, superadditivity in its second argument.

Proof of Theorem 2. The proof relies on the following auxiliary lemma.

Lemma 1. Let $N \in \mathscr{N}$ and let $(d ; E)$ and $\left(d^{\prime} ; E\right)$ be two bankruptcy problems in $B^{N}$ such that $d_{k}=d_{k}^{\prime}$ for all $k \neq i, j$ and $d_{i}^{\prime}=d_{j}^{\prime}=\left(d_{i}+d_{j}\right) / 2$. Any non manipulable by splitting parametric rule satisfies that for all $\lambda^{\prime} \in \Lambda\left(d^{\prime}\right)$ there exists $\lambda \in \Lambda(d)$ such that $\lambda \leq \lambda^{\prime}$.

Proof. Assume first $n=2$. Thus, let $(d ; E)$ and $\left(d^{\prime} ; E\right)$ be two bankruptcy problems such that $d=\left(d_{1}, d_{2}\right)$ and $d^{\prime}=\left(\left(d_{1}+d_{2}\right) / 2,\left(d_{1}+d_{2}\right) / 2\right)$. Let $\lambda$ be the smallest value in $\Lambda(d)$, and let $\lambda^{\prime} \in \Lambda\left(d^{\prime}\right)$. Assume, by way of contradiction, $\lambda>\lambda^{\prime}$.

By the (weak) monotonicity of $h$ and since $\lambda^{\prime} \notin \Lambda(d)$ (Notice that $\lambda>\lambda^{\prime}$ implies that $\left.\lambda^{\prime} \notin \Lambda(d)\right)$ the following inequality must hold:

$$
h\left(\lambda^{\prime}, d_{1}\right)+h\left(\lambda^{\prime}, d_{2}\right)<h\left(\lambda^{\prime}, \frac{d_{1}+d_{2}}{2}\right)+h\left(\lambda^{\prime}, \frac{d_{1}+d_{2}}{2}\right) .
$$

Let us denote $d_{1}$ by $x,\left(d_{1}+d_{2}\right) / 2$ by $y$ and, finally, $\left(d_{2}-d_{1}\right) / 2$ by $z$. Inequality above can be rewritten as:

$$
h\left(\lambda^{\prime}, y+z\right)-h\left(\lambda^{\prime}, y\right)<h\left(\lambda^{\prime}, x+z\right)-h\left(\lambda^{\prime}, x\right),
$$

where $x, y, z \geq 0$ and $x<y$. Now, appealing to Proposition 8 in Sharkey and Telser [10] $h$ has to satisfy: 


$$
h\left(\lambda^{\prime}, x\right)-h\left(\lambda^{\prime}, x-z\right)<\frac{h\left(\lambda^{\prime}, x\right)-h\left(\lambda^{\prime}, x-\theta z\right)}{\theta},
$$

where $x-\theta z>0$ and $\theta \geq 1$.

As $x-\theta z \rightarrow 0^{+}, h\left(\lambda^{\prime}, x-\theta z\right) \rightarrow 0^{+}$thus inequality (3) becomes $h\left(\lambda^{\prime}, x\right)<$ $\frac{x}{x-z} h\left(\lambda^{\prime}, x-z\right)$, contradicting that $h$ is a NAS rule. ${ }^{9}$ Therefore $\lambda$ cannot be greater than $\lambda^{\prime}$.

Let now $n>2$. Let $(d ; E)$ and $\left(d^{\prime} ; E\right)$ be two bankruptcy problems in $B^{N}$ such that $d_{k}=d_{k}^{\prime}$ for all $k \neq i, j$ and $d_{i}^{\prime}=d_{j}^{\prime}=\left(d_{i}+d_{j}\right) / 2$, and let $\lambda \in \Lambda(d)$ and $\lambda^{\prime} \in \Lambda\left(d^{\prime}\right)$. Consistency implies that the shares of claimants $i$ and $j$ when they face the problem $(d ; E)$ coincide with their shares when they face the reduced problem $\left(d_{M} ; A\right)$, where $A$ equals $E-\sum_{k \neq i, j} h\left(\lambda, d_{k}\right)$ and where $d_{M}=\left(d_{i}, d_{j}\right)$; analogously, the shares of claimants $i$ and $j$ when they face the problem $\left(d^{\prime} ; E\right)$ coincide with their shares when they face the reduced problem $\left(d_{M}^{\prime} ; B\right)$, where $d_{M}^{\prime}=\left(\left(d_{i}+d_{j}\right) / 2,\left(d_{i}+d_{j}\right) / 2\right)$ and $B=E-\sum_{k \neq i, j} h\left(\lambda^{\prime}, d_{k}\right)$, for any $\lambda \in \Lambda(d)$ and $\lambda^{\prime} \in \Lambda\left(d^{\prime}\right)$. Obviously any $\lambda \in \Lambda(d)$ also belongs to $\Lambda\left(\left(d_{M} ; A\right)\right)$, and any $\lambda^{\prime} \in \Lambda\left(d^{\prime}\right)$ belongs to $\Lambda\left(\left(d_{M}^{\prime} ; B\right)\right)$ as well. Thus, we have:

$$
\begin{aligned}
h\left(\lambda, d_{i}\right)+h\left(\lambda, d_{j}\right) & =A \\
h\left(\lambda^{\prime}, \frac{d_{i}+d_{j}}{2}\right)+h\left(\lambda^{\prime}, \frac{d_{i}+d_{j}}{2}\right) & =B .
\end{aligned}
$$

For $A \leq B$ we have that $\lambda \leq \lambda^{\prime}$ since this is the case for $n=2$. For $A>B$ the result holds since $A>B$ implies $\sum_{k \neq i, j} h\left(\lambda, d_{k}\right)<\sum_{k \neq i, j} h\left(\lambda^{\prime}, d_{k}\right)$ which implies $\lambda<\lambda^{\prime}$.

Thus for any number of creditors the statement holds.

Proof of the only if part of (a) in Theorem 2. From Young's result we know that any continuous, symmetric and consistent rule is a parametric rule relative to a function $h$. We now show that if $h$ is non manipulable by splitting, then $h$ is convex in its second argument for each value of the parameter $\lambda$.

To prove this result, since $h$ is continuous on $(0, \infty)$, it suffices to show that for all $d_{i}, d_{j}$ and for fixed $\lambda$ :

$$
h\left(\lambda, \frac{d_{i}+d_{j}}{2}\right) \leq \frac{h\left(\lambda, d_{i}\right)+h\left(\lambda, d_{j}\right)}{2} .
$$

Let $N \in \mathscr{N}$ and let $(d ; E)$ and $\left(d^{\prime} ; E\right)$ be two bankruptcy problem in $B^{N}$ such that $d_{k}=d_{k}^{\prime}$ for all $k \neq i, j$ and $d_{i}^{\prime}=d_{j}^{\prime}=\left(d_{i}+d_{j}\right) / 2$. Let $\lambda \in \Lambda(d)$. Because of Lemma 1 we know that there exists $\lambda^{\prime} \in \Lambda\left(d^{\prime}\right)$ such that $\lambda \leq \lambda^{\prime}$.

Since $\lambda \leq \lambda^{\prime}$ we have $\sum_{k \neq i, j} h\left(\lambda, d_{k}\right) \leq \sum_{k \neq i, j} h\left(\lambda^{\prime}, d_{k}\right)$. This implies $h\left(\lambda, d_{i}\right)+h\left(\lambda, d_{j}\right) \geq 2 h\left(\lambda^{\prime},\left(d_{i}+d_{j}\right) / 2\right) \geq 2 h\left(\lambda,\left(d_{i}+d_{j}\right) / 2\right)$, where the last inequality follows from the monotonicity of $h$ in $\lambda$.

Thus $h\left(\lambda, \frac{d_{i}+d_{j}}{2}\right) \leq \frac{h\left(\lambda, d_{i}\right)+h\left(\lambda, d_{j}\right)}{2}$.

\footnotetext{
9 A NAS rule is necessarily superadditive, and thus, appealing to Theorem 143 in Rosenbaum [9], it must satisfy weak superhomogeneity as well, (see also Newman[7])
} 
Proof of the if part of (a) in Theorem 2. We now show that if a parametric rule is convex in its second argument then it is a no advantageous splitting rule. Note that Young's theorem implies that it is also continuous, symmetric and consistent.

To prove the statement we only need to show that the convexity of $h$ in its second argument implies that for all $N, M$ in $\mathscr{N}$, with $N \supset M$, if $(d ; E) \in B^{N}$ and $\left(d^{\prime}, E\right) \in B^{M}$ are such that there exists $i \in M$ with $d_{i}^{\prime}=d_{i}+\sum_{j \in N \backslash M} d_{j}$ and with $d_{k}^{\prime}=d_{k}$ for all $k \in M \backslash\{i\}$, then $\lambda \geq \lambda^{\prime}$ holds for any $\lambda \in \Lambda(d)$ and $\lambda^{\prime} \in \Lambda\left(d^{\prime}\right)$.

Since $h$ is a parametric rule it satisfies that $h(\lambda, 0)=0$ for all $\lambda$. Thus for $\lambda$ fixed, Theorem 1.4.3. in Rosenbaum[9] ensures that $h$ is a superadditive in its second argument function since it is convex and it satisfies $h(\lambda, 0)=0$. Now, $d_{i}^{\prime}=d_{i}+\sum_{j \in N \backslash M} d_{j}$ and the superadditivity of $h$ imply

$$
h\left(\lambda, d_{i}^{\prime}\right) \geq h\left(\lambda, d_{i}\right)+\sum_{j \in N \backslash M} h\left(\lambda, d_{j}\right)
$$

By adding $\sum_{k \in M \backslash\{i\}} h\left(\lambda, d_{k}\right)$ to both sides of (9), budget balance yields

$$
h\left(\lambda, d_{i}^{\prime}\right)+\sum_{k \in M \backslash\{i\}} h\left(\lambda, d_{k}\right) \geq E .
$$

The left hand side of (10) is equal to $E$ at $\lambda^{\prime}$ (recall that $d_{k}=d_{k}^{\prime}$ for all $k \in$ $M \backslash\{i\}$ ). Thus the (weak) increasing monotonicity of $h$ in its first argument allows to conclude that $\lambda \geq \lambda^{\prime}$.

Proof of the only if part of $(b)$ in Theorem 2. Let $h$ be a NAM parametric rule. If $h$ is parametric so is its dual. Let $g$ be the parametric rule which is dual of $h$. By the result in Proposition $1 \mathrm{~g}$ is a NAS rule, and hence, by part (a) in theorem $2, g\left(\lambda, d_{i}\right)$ is convex in $d_{i}$.

Since $h$ is the addition of two concave in $d_{i}$ functions, recall that $h\left(\lambda, d_{i}\right)=$ $d_{i}-g\left(\lambda, d_{i}\right)$, it has to be concave in $d_{i}$, as we claimed.

Proof of the if part of (b) in Theorem 2. Concavity of $h$ in $d_{i}$ implies that its dual is convex in $d_{i}$ and so a NAS rule as we have shown. If the dual is a NAS rule, Proposition 1 implies that $h$ is a no advantageous merging rule.

Proof of (a) in Theorem 3. The proof relies on the following auxiliary lemma.

Lemma 2. If $h$ is a non advantageous splitting parametric rule $X$ then any pseudo-inverse of $h$ is an almost concave function. ${ }^{10}$

${ }^{10}$ From Kuczma [3] it is known that a function $f: I \times I \rightarrow \mathscr{R}$ is almost concave if

$$
f\left(\frac{x+y}{2}\right) \geq \frac{1}{2}[f(x)+f(y)],
$$

holds for all $(x, y) \in I \times I$ except on a set $M \subseteq I \times I$ of Lebesgue measure zero, where $I$ is any open interval of the real line Kuczma proves that an almost concave function is equal to a concave function almost everywhere 
Proof. Let $N \in \mathscr{N}$ and let $(d ; E)$ and $\left(d^{\prime} ; E\right)$ be two bankruptcy problems in $B^{N}$ such that $d_{k}=d_{k}^{\prime}$ for all $k \neq i, j$ and $d_{i}^{\prime}=d_{j}^{\prime}=\left(d_{i}+d_{j}\right) / 2$. In lemma 1 we showed that any no advantageous splitting parametric rule satisfies that for all $\lambda^{\prime} \in \Lambda\left(d^{\prime}\right)$ there exists $\lambda \in \Lambda(d)$ such that $\lambda \leq \lambda^{\prime}$. Hence $h\left(\lambda, d_{i}\right)+$ $h\left(\lambda, d_{j}\right) \geq 2 h\left(\lambda^{\prime},\left(d_{i}+d_{j}\right) / 2\right)$, for $\lambda \leq \lambda^{\prime}$. Let $x=h\left(\lambda, d_{i}\right), y=h\left(\lambda, d_{j}\right)$, and $z=h\left(\lambda^{\prime},\left(d_{i}+d_{j}\right) / 2\right)$. Denoting by $f$ any pseudo-inverse of $h$, we have that for the points where $f$ is continuous $\lambda=f\left(x, d_{i}\right)=f\left(y, d_{j}\right)$ and $\lambda^{\prime}=f\left(z,\left(d_{i}+d_{j}\right) / 2\right)$. Since $z \leq(x+y) / 2$ there is $\delta \geq 0$, such that $z=(x+y) / 2-\delta$. Thus $\lambda \leq \lambda^{\prime}$ implies:

$$
\frac{f\left(x, d_{i}\right)+f\left(y, d_{j}\right)}{2} \leq f\left(\frac{x+y}{2}-\delta, \frac{d_{i}+d_{j}}{2}\right) \leq f\left(\frac{x+y}{2}, \frac{d_{i}+d_{j}}{2}\right),
$$

where the last inequality follows from the monotonicity of $f$ in its first argument.

Hence for any $x_{i} \in(0, \infty)$, and any $d_{i} \in(0, \infty)$ except at the countable set of points where $f$ is discontinuous, -a set with Lebesgue measure zero-, $f$ is an almost concave function.

Proof of (a) in Theorem 3. If $h$ minimizes an objective function that takes the form we claim then it is straightforward to check that it satisfies convexity in its second argument. To prove the converse statement let $h$ be a no advantageous splitting parametric rule. From Theorem 2 in Young [13] it is known that $h$ is solution of the following optimization problem:

$\min \sum_{i=1}^{n} H\left(x_{i}, d_{i}\right)$ subject to $\sum_{i=1}^{n} x_{i}=E$ and $0 \leq x_{i} \leq d_{i}$.

As solution to the above program $h$ has to satisfy the Kuhn-Tucker conditions associated with the optimization program. Three cases have to be considered:

1. The constraints $0 \leq x_{i} \leq d_{i}$ are never binding at the optimum.

If the constraints are never binding then $f$ is a inverse of $h$. It will be strictly concave whenever $h$ is strictly convex. Moreover it satisfies $0<f\left(x_{i}, d_{i}\right)=$ $\lambda<1$. If $\lambda=1$ then $h\left(1, d_{i}\right)=d_{i}$ for all $d_{i}$. Thus $h$ must be linear in $d_{i}$ for $\lambda=1$ (similarly for $\lambda=0$ ). Continuity implies that $h$ must be linear in $d_{i}$ for all $\lambda$. Thus we must have $h\left(\lambda, d_{i}\right)=d_{i} \alpha(\lambda)$ where $\lambda=\alpha^{-1}\left(x_{i} / d_{i}\right)$. Since $\alpha(\lambda)$ has to be continuous, strictly monotone increasing in $\lambda$, and with $\alpha(0)=0$ and $\alpha(1)=1$, then we can take $\alpha(\lambda)=\lambda$. Thus $x_{i}=h\left(\lambda, d_{i}\right)=d_{i} \lambda$, and $\lambda=f\left(x_{i}, d_{i}\right)=x_{i} / d_{i}$. Consequently, $H\left(x_{i}, d_{i}\right)=\int_{0}^{x_{i}} f\left(a, d_{i}\right) d a$ will take the form $H\left(x_{i}, d_{i}\right)=x_{i}^{2} / 2 d_{i}$.

2. Some of the Kuhn-Tucker coefficients associated with the constraints $x_{i} \leq d_{i}$ are strictly positive.

For such $i$ we will have $x_{i}=d_{i}$. Hence $x_{i}=h\left(\lambda, d_{i}\right)$ will take the form $x_{i}=\min \left\{d_{i}, H_{x}^{-1}\left(\lambda, d_{i}\right)\right\}$. Since $H_{x}^{-1}\left(\lambda, d_{i}\right)$ is almost concave , and since the minimum of two concave functions is a concave function as well, $x_{i}=h\left(\lambda, d_{i}\right)$ will be concave in $d_{i}$. Therefore 2 . can never be the case.

3. Some of the Kuhn-Tucker coefficients associated with the constraints $0 \leq x_{i}$ are strictly positive.

For such $i$ we will have $x_{i}=0$. Hence $x_{i}=h\left(\lambda, d_{i}\right)$ will take the form $x_{i}=\max \left\{0, H_{x}^{-1}\left(\lambda, d_{i}\right)\right\}$. For the maximum to be convex in $d_{i}, H_{x}^{-1}\left(\lambda, d_{i}\right)$ 
has to be linear in $d_{i}$. Since the constrains are binding for at least one $i$, then $H_{x}^{-1}\left(\lambda, d_{i}\right)<0$ for that $i$. Taking into account the definition of parametric rules we can write $h\left(\lambda, d_{i}\right)=d_{i}-\gamma(\lambda)$ for some $\gamma($.) weakly monotone decreasing in $\lambda$. This yields $\lambda=f\left(x_{i}, d_{i}\right)=\gamma^{-1}\left(d_{i}-x_{i}\right)$. Thus $H\left(x_{i}, d_{i}\right)=$ $\int_{0}^{x_{i}} f\left(a, d_{i}\right) d a$ will take the form $H\left(x_{i}, d_{i}\right)=-U\left(d_{i}-x_{i}\right)+C$, where $U$ has to be a strictly convex function. Since $H\left(0, d_{i}\right)$ has to be equal to 0 for all $d_{i}$, then $C$ has to be equal to $U\left(d_{i}\right)$. Thus $H\left(x_{i}, d_{i}\right)=U\left(d_{i}\right)-U\left(d_{i}-x_{i}\right)$. From the three cases we have studied we conclude that $H\left(x_{i}, d_{i}\right)$ will take the form $H\left(x_{i}, d_{i}\right)=U\left(d_{i}\right)-U\left(d_{i}-x_{i}\right)$ for any NAS rule but the proportional one.

Proof of (b) in Theorem 3. Let $h$ be a NAM parametric rule, but the proportional. Theorem 2 (b) states that $h$ is concave in $d_{i}$ and Proposition 1 states that $h$ is the dual of some NAS parametric rule. Thus $\bar{x}_{i}=h\left(\lambda, d_{i}\right)=d_{i}-x_{i}=d_{i}-g\left(\lambda, d_{i}\right)$, where $h$ is the dual of $g$. From the previous theorem we know that $g\left(\lambda, d_{i}\right)$ can be constructed by solving the following program:

$\min \sum_{i=1}^{n} U\left(d_{i}\right)-U\left(d_{i}-x_{i}\right)$ subject to $\sum_{i=1}^{n} x_{i}=E$ and $0 \leq x_{i} \leq d_{i}$,

where $U\left(d_{i}\right)-U\left(d_{i}-x_{i}\right)=\int_{0}^{x_{i}} s\left(a, d_{i}\right) d a$, where $s$ is a pseudo-inverse of $g$. Denoting by $f$ to the pseudo inverse of $h$, the dual relationship yields:

$\lambda=s\left(x_{i}, d_{i}\right)=s\left(d_{i}-\bar{x}_{i}, d_{i}\right)$, and $U\left(d_{i}\right)-U\left(d_{i}-x_{i}\right)=\int_{0}^{x_{i}} s\left(a, d_{i}\right) d a=$ $\int_{0}^{x_{i}} f\left(d_{i}-a, d_{i}\right) d a$.

Equalities above imply

$\int_{0}^{x_{i}} f\left(a, d_{i}\right) d a=\int_{0}^{x_{i}} s\left(d_{i}-a, d_{i}\right) d a=U\left(d_{i}\right)+U\left(d_{i}-\left(d_{i}-x_{i}\right)\right)+C=U\left(d_{i}\right)+$ $U\left(x_{i}\right)+C$,

for some $U$ strictly convex. Now $H\left(0, d_{i}\right)=0$ implies $C=-U\left(d_{i}\right)$.

Finally, since the proportional rule is self-dual then part $(a)$ of this theorem implies $H\left(x_{i}, d_{i}\right)=x_{i}^{2} / 2 d_{i}$ for the proportional rule.

\section{References}

1 Aumann, R J , Maschler, M (1985) Game Theoretic Analysis of a Bankruptcy Problem from the Talmud Journal of Economic Theory 36: 195-213

2 Chun, Y (1988) The Proportional Solution for Rights Problems Mathematical Social Science 15: 231-246

3 Kuczma, M E (1970) Almost Convex Functions Colloquia Mathematics 21: 279-284

4 Moulin, H (1985) The separability Axiom and Equal-sharing Methods Journal of Economic Theory 36: 120-148

5 Moulin, H (1987) Equal or Proportional Division of a Surplus, and Other Methods International Journal of Game Theory 16: 161-186

6 Moulin, H (1988) Axioms of Cooperative Decision Making Cambridge University Press, Cambridge, England

7 Newman, P (1969) Some Properties of Concave Functions Journal of Economic Theory 1: 291-314

8 O'Neill, B (1982) A Problem of Right Arbitration in the Talmud Mathematical Social Science 2: $345-371$

9 Rosenbaum, R (1950) Subadditive functions Duke Mathematical Journal 17: 227-247

10 Sharkey, W, Telser, L (1978) Supportable Cost Functions for the Multiproduct Firm Journal of Economic Theory 18: 23-37

11 Thomson, W (1996) Consistent Allocation Rules Rochester Center for Economic Research, Mimeo 
12. Thomson, W. (1995) Axiomatic Analyses of Bankruptcy and Taxation Problems: A Survey. Rochester Center for Economic Research, W.P. 413

13. Young, H. P. (1987) On Dividing an Amount in Accordance to Individual Claims or Liabilities. Mathematics of Operations Research 12: 397-414

14. Young, H. P. (1988) Distributive Justice in Taxation. Journal of Economic Theory 32: 321-335 